DOI:http://dx.doi.org/10.18273/revint.v35n1-2017006

\title{
Geoestadística aplicada a series de tiempo autorregresivas: un estudio de simulación
}

\author{
Ramón Giraldo $^{a *}$, Óscar PaCheco $^{b}$, Astrid Orozco $^{c}$ \\ ${ }^{a}$ Departamento de Estadística, Universidad Nacional de Colombia, Bogotá, \\ Colombia. \\ ${ }^{b}$ Departamento de Matemáticas, Escuela Colombiana de Ingeniería Julio Garavito, \\ Bogotá, Colombia. \\ ${ }^{c}$ Turkish Petroleum Company, Bogotá, Colombia.
}

Resumen. La geoestadística puede usarse como método de predicción de datos faltantes en series temporales. El procedimiento se basa en el estudio de la estructura de autocorrelación temporal de la serie de tiempo por medio de la función de variograma, que es estimada por mínimos cuadrados (geoestadística clásica) o por máxima verosimilitud (geoestadística basada en modelo), y en usar posteriormente Kriging para hacer predicción de los valores faltantes. En este trabajo se comparan a través de simulación las dos aproximaciones (geoestadística clásica y basada en modelo) en el contexto de series temporales autorregresivas.

Palabras clave: Autocorrelación, Datos faltantes, Kriging, Predicción, Semivariograma, Series de tiempo, Variograma.

MSC2010: 60G15, 62M10, 62M20, 62M30, 86A32.

\section{Geostatistics applied to autorregresive time series: A simulation study}

\begin{abstract}
Geostatistics can be used as a method for predicting missing data in time series. The procedure is based on estimating the temporal autocorrelation structure by means of the semivariance function, by least squares (classical geostatistics) or maximum likelihood (model-based geostatistics), and posteriorly using Kriging for doing prediction of missing data in the time series. In this work we compare classical and model-based geoestatistics in the context of time series using simulated autorregresive time series.

Keywords: Autocorrelation, Kriging, Prediction, Missing values, Semivariogram, Time series, Variogram.

\footnotetext{
${ }^{*}$ E-mail: rgiraldoh@unal.edu.co

Recibido: 28 de abril de 2016, Aceptado: 31 de mayo de 2017.

Para citar este artículo: R. Giraldo, Ó. Pacheco, A. Orozco, Geoestadística aplicada a series de tiempo autorregresivas: un estudio de simulación, Rev. Integr. Temas Mat. 35 (2017), No. 1, 83-102.
} 


\section{Introducción}

Un campo de investigación en series de tiempo es la predicción de valores faltantes. Existen diversas aproximaciones para dar solución al problema que presentan los vacíos de información en series temporales [9]. Algunas tratan este tema como la estimación de los parámetros de un modelo en presencia de datos faltantes y lo resuelven por máxima verosimilitud (MV). Otras hacen la estimación de los valores faltantes, considerándolos como predicciones de variables aleatorias dado un conjunto de datos y posteriormente estiman los parámetros del modelo con la serie resultante. La diferencia entre estos enfoques es presentada en [12]. Cuando la solución se busca desde el enfoque de predicción, se acude a herramientas tradicionales en el estudio de series temporales en las que juega un papel importante el estudio de la estructura de autocorrelación de la serie. Este hecho sugiere la posibilidad de utilizar el análisis geoestadístico [2] en la solución del problema en consideración, puesto que los dos pilares básicos de dicha metodología son el estudio de la estructura de autocorrelación de datos (espacial o temporal) a través de la función de variograma, y su uso en la predicción de los valores de la variable en sitios o periodos de tiempo no observados. El variograma ha sido empleado en el contexto de series de tiempo (especialmente por la comunidad geoestadística). Varios autores han utilizado la geoestadística para interpolar series temporales con dominio de tiempo discreto y que pueden ser no estacionarias. En este sentido [7] muestra la conveniencia de considerar el variograma (en vez de la función de covarianza) en series de tiempo no estacionarias y lo aplica en la modelación de una serie de tiempo de temperatura; [10] trabaja en la construcción de modelos de series de tiempo estacionarias a través del variograma; [3] hace una revisión del uso variograma en series temporales para la identificación de tendencia, predicción de valores faltantes, pronóstico y estimación de la función de distribución; [5] y [6] hacen aplicaciones del análisis geoestadístico en la modelación de series temporales. En el primer caso a dos series radiométricas ambientales, y en el segundo a una serie de precipitación.

En los artículos mencionados se resalta la ventaja de trabajar con el variograma en vez de la covarianza (correlación), especialmente con procesos no estacionarios. En estos se acude al enfoque clásico del análisis geoestadístico basado en la estimación de la función de variograma por mínimos cuadrados (MC) y en la predicción por Kriging [16]. En este trabajo no se hace una discusión respecto a la validez del uso de la geoestadística en series temporales, considerando que este tema ha sido ampliamente estudiado. El objetivo es comparar en el contexto de series la aproximación clásica con la basada en modelo [4], en la cual se hace estimación por MV de la función de variograma.

Infortunadamente las propiedades de los estimadores de los parámetros de los modelos válidos de variograma no están claramente definidas (ver [2, Sección 2.6.3]). Tomar una decisión al respecto de cuál metodología produce mejores resultados puede ser una tarea difícil desde un enfoque teórico. Por ello en el artículo se acude a la simulación como herramienta de comparación. Existen muchos procedimientos para el análisis de las series temporales, entre los que se encuentran los métodos de suavizado, de descomposición, los modelos autorregresivos condicionales heterocedásticos (ARCH), autorregresivos integrados de media móvil (ARIMA), el análisis espectral y los modelos autorregresivos en tiempo continuo (CAR). Dentro de los ARIMA, los más sencillos son los modelos autorregresivos (AR). Considerar el efecto de los métodos de estimación en todos estos haría muy extenso el estudio. Por simplicidad en la presentación se decidió trabajar con

[Revista Integración, temas de matemáticas 
los modelos AR. En concreto se determina que método de estimación del variograma (clásico o basado en modelo) es más favorable cuando se estudian series de tiempo que son realizaciones de este tipo de modelos [1].

El artículo está organizado de la siguiente forma. En la primera parte se revisan los conceptos básicos de series y de geoestadística (Secciones 2 y 3). Para hacer una comparación del efecto de los métodos de estimación del semivariograma en la predicción de valores faltantes en series de tiempo autorregresivas se lleva a cabo un análisis de simulación (Sección 4). El artículo termina con unas conclusiones y propuestas de trabajo futuro.

\section{Series de tiempo}

A continuación se hace una revisión de las propiedades de los modelos AR que serán posteriormente considerados en la Sección 4.

\subsection{Modelos AR}

Sea $\{Z(t), t \in T\}$ una serie de tiempo estacionaria con media $\mu$ y varianza $\sigma^{2}$. El modelo autorregresivo de orden uno AR (1) se define como [1]

$$
Z(t)=c+\phi_{1} Z(t-1)+a(t),
$$

donde $a(t)$ es un ruido con media cero y varianza $\sigma_{a}^{2}$ y $c$ y $\phi$ son las constantes a estimar. Se puede mostrar que la media y la varianza están dadas por

$$
\begin{aligned}
c & =\mu-\phi_{1} \mu, \\
\sigma^{2} & =\frac{\sigma_{a}^{2}}{1-\phi_{1}^{2}} .
\end{aligned}
$$

Si $\tilde{Z}(t)=Z(t)-\mu$, entonces el modelo (1) puede escribirse como

$$
\tilde{Z}(t)=\phi_{1} \tilde{Z}(t-1)+a(t) \text {. }
$$

Las funciones de covarianza y correlación del proceso AR (1) están dadas, respectivamente, por

$$
\begin{aligned}
\mathbb{C}(Z(t), Z(t-h)) & =\phi_{1} \mathbb{C}(Z(t), Z(t-(h-1)), h \in \mathbb{Z}, \\
\rho(Z(t), Z(t-h)) & =\phi^{h}, h \in \mathbb{Z} .
\end{aligned}
$$

De manera análoga, el modelo AR (2) y su varianza, covarianza y correlación son dados [1]:

$$
\begin{aligned}
\tilde{Z}(t) & =\phi_{1} \tilde{Z}(t-1)+\phi_{2} \tilde{Z}(t-2)+a(t), \\
\mathbb{C}(Z(t), Z(t-h)) & =\sum_{j=1}^{2} \phi_{j} \mathbb{C}(Z(t), Z(t-(h-j)), h \in \mathbb{Z}, \\
\rho(Z(t), Z(t-h)) & =\sum_{j=1}^{2} \phi_{j} \rho(Z(t), Z(t-(h-j)), h \in \mathbb{Z}, \\
\sigma^{2} & =\frac{\left(1-\phi_{2}\right) \sigma_{a}^{2}}{\left(1+\phi_{2}\right)\left(1-\phi_{1}-\phi_{2}\right)\left(1+\phi_{1}-\phi_{2}\right)} .
\end{aligned}
$$

Vol. 35, No. 1, 2017] 
Los coeficientes en este caso tienen las restricciones $\left|\phi_{2}\right|<1,\left(\phi_{1}+\phi_{2}\right)<1$ y $\left(\phi_{2}-\phi_{1}\right)<1$. Generalizando los modelos (3) y (5) al caso de $p$ periodos de tiempo, se tiene el modelo AR (P) dado por

$$
\tilde{Z}(t)=\phi_{1} \tilde{Z}(t-1)+\phi_{2} \tilde{Z}(t-2)+\cdots+\phi_{p} \tilde{Z}(t-p)+a(t) .
$$

Usando el operador de retardo $B$ dado por $B Z(t)=Z(t-1), \cdots, B^{k} Z(t)=Z(t-k)$, el modelo (8) se puede escribir como $\left(1-\phi_{1} B-\phi_{2} B^{2}-\cdots \phi_{p} B^{p}\right) \tilde{Z}(t)=a(t)$. En este modelo se tiene la restricción $\left|\phi_{1}+\phi_{2}+\cdots+\phi_{p}\right|<1$. La expresión general para la varianza del modelo AR (P) está dada por [1]

$$
\sigma^{2}=\sum_{i=1}^{p} \phi_{i} \mathbb{C}(Z(t), Z(t-i))+\sigma_{a}^{2}
$$

con

$$
\mathbb{C}(Z(t), Z(t-i))=\sum_{j=1}^{p} \phi_{j} \mathbb{C}(Z(t)-Z(t-(i-j)) .
$$

\section{Geoestadística}

En un análisis geoestadístico deben cumplirse dos etapas. En la primera se estudia la dependencia (espacial y/o temporal) a través de la estimación de la función de variograma $2 \gamma(h)$ y posteriormente se emplea un predictor Kriging [2] para hacer predicción en sitios de una región o en puntos del tiempo no observados. La función de semivariograma (mitad del variograma) y su relación con la de covarianza y de correlación está dada por [16]:

$$
\begin{aligned}
\gamma(h) & =\frac{1}{2} \mathbb{E}(Z(t)-Z(t+h))^{2}, \\
C(h) & =\sigma^{2}-\gamma(h), \\
\rho(h) & =\frac{C(h)}{\sigma^{2}} .
\end{aligned}
$$

La estimación de la función de semivariograma (ecuación 9) puede hacerse por MC o MV [4]. Los modelos válidos de semivariograma son aquellos que garantizan que $\sum_{i=1}^{k} \sum_{j=1}^{k} a_{i} a_{j} 2 \gamma\left(t_{i}, t_{j}\right) \leq 0$. Una revisión de modelos que cumplen con esta condición puede hacerse en [16]. Los modelos de semivariograma más ampliamente usados en la práctica son exponencial, esférico, gaussiano y de Matérn (ver [16]). En este trabajo se usa el exponencial, que es definido como

$$
\gamma(h ; \theta)=\tau^{2}+\sigma^{2}(1-\exp (-h / \phi)),
$$

donde $\tau^{2}$ (efecto nugget) [16] es una discontinuidad del semivariograma en $h=0$ [16], $\sigma^{2}=\mathbb{V}(Z(t))$ y $\phi$ (rango) es la distancia hasta la cual hay correlación temporal. En el resto del trabajo se asume $\tau^{2}=0$. A continuación se detallan los dos métodos de estimación antes mencionados.

[Revista Integración, temas de matemáticas 


\subsection{Estimación por mínimos cuadrados}

Usando el método de momentos, el estimador de la función de semivariograma (ecuación 9) está dado por el semivariograma experimental [2] definido como

$$
\bar{\gamma}(h)=\frac{1}{2 n(h)} \sum_{n(h)}(Z(t+h)-Z(t))^{2}
$$

donde $Z(t)$ es el valor de la variable en el periodo de tiempo $t, Z(t+h)$ es otro valor observado separado del anterior por un intervalo de tiempo $h$ y $n(h)$ es el número de parejas de periodos de tiempo que se encuentran separados por una distancia $h$. Una vez obtenida la función (11), para diferentes valores de $h$, se emplea regresión no lineal para ajustar por MC (ordinarios o ponderados) un modelo teórico de semivarianza al dispersograma dado por las parejas $(h, \bar{\gamma}(h))$, obteniéndose así la estimación $\hat{\gamma}(h)$ de la función dada en la ecuación (9). Los parámetros de interés se estiman por MC ordinarios (MCO) y MC ponderados (MCP) minimizando, respectivamente, las siguientes sumas de cuadrados de errores:

$$
\begin{aligned}
& S C E_{1}=\sum_{k}\left(\bar{\gamma}\left(h_{k}\right)-\gamma\left(h_{k} ; \theta\right)\right)^{2}, \\
& S C E_{2}=\sum_{k} n_{k}\left(\bar{\gamma}\left(h_{k}\right)-\gamma\left(h_{k} ; \theta\right)\right)^{2},
\end{aligned}
$$

donde $\bar{\gamma}\left(h_{k}\right)$ está dado por (11), $\gamma\left(h_{k} ; \theta\right)$ corresponde a un modelo de semivariograma teórico (exponencial, esférico, gaussiano, de Matérn, etc.) evaluado para la distancia temporal $h_{k}$; con $\theta$ el vector de parámetros a estimar (por ejemplo $\theta=\left(\tau^{2}, \sigma^{2}, \phi\right)$ en el caso del modelo exponencial) y $n_{k}$ es el número de parejas de periodos de tiempo separados por una distancia $h_{k}$.

\subsection{Estimación por máxima verosimilitud}

Se supone que el proceso estocástico $\{Z(t), t \in T\}$ es gaussiano, es decir que si se tienen $n$ periodos de tiempo, el vector $Z(t)=\left(Z\left(t_{1}\right), Z\left(t_{2}\right), \cdots, Z\left(t_{n}\right)\right)^{T}$ sigue una distribución normal multivariada $Z(t) \sim N M V(\mu, \Sigma(\theta))$, donde $\theta=\left(\tau^{2}, \sigma^{2}, \phi\right), \Sigma(\theta)=$ $\left[\tau^{2} I_{n \times n}+\sigma^{2} R(\phi)\right]$, con $I_{n \times n}$ la matriz identidad y $R(\phi)_{n \times n}$ es la matriz de correlación cuyo elemento $i j$-ésimo es $r_{i j}=\rho\left(\left\|t_{i}-t_{j}\right\|, \phi\right), i, j=1, \cdots, n$. De acuerdo con lo anterior, la función de verosimilitud está dada por

$$
L(\mu, \theta)=\frac{1}{(2 \pi)^{n / 2}|\Sigma(\theta)|^{1 / 2}} \exp \left(-\frac{1}{2}(Z(t)-\mu)^{T} \Sigma(\theta)^{-1}(Z(t)-\mu)\right) .
$$

Tomando logaritmo en (12) se obtiene (sin la constante) la función a maximizar:

$$
\ell(\mu, \theta)=-\frac{1}{2}\left(|\Sigma(\theta)|+(Z(t)-\mu)^{T} \Sigma(\theta)^{-1}(Z(t)-\mu)\right) .
$$

Dado $\theta$, el estimador máximo verosímil de $\mu$ es

$$
\hat{\mu}(\theta)=\left(\mathbf{1}^{T} \Sigma(\theta)^{-1} \mathbf{1}\right)^{-1}\left(\mathbf{1}^{T} \Sigma(\theta)^{-1} Z(t)\right) .
$$

Vol. 35, No. 1, 2017] 
Reemplazando (14) en (13) se establece la función log-verosímil, que es maximizada por métodos numéricos (por ejemplo, Newton-Raphson o el algoritmo de Fisher). De esta forma se obtiene $\hat{\theta}$, y por consiguiente la estimación de la función de semivariograma $\gamma(h ; \hat{\theta})$.

\subsection{Kriging}

Una vez estimado el modelo teórico de semivarianza (equivalentemente el de covarianza o de correlación) es posible realizar la predicción en puntos del tiempo no muestreados, empleando un predictor Kriging [2]. Si el proceso es estacionario cada observación faltante es predicha mediante

$$
\hat{Z}\left(t_{p}\right)=\sum_{i=1}^{n} \lambda_{i} Z\left(t_{i}\right)
$$

con $p$ un punto del tiempo donde no hay observación. El predictor (15) se denomina Kriging ordinario. Poniendo la restricción $\sum_{i=1}^{n} \lambda_{i}=1$, este es insesgado [2]. Los pesos $\lambda_{i}$ se obtienen minimizando la varianza del error de predicción $\mathbb{V}\left(\hat{Z}\left(t_{p}\right)-Z\left(t_{p}\right)\right)$ sujeto a la restricción de insesgadez, es decir se obtienen $\operatorname{los} \lambda_{i}$ de tal manera que el predictor (15) sea el mejor predictor lineal insesgado. La varianza de predicción en cada punto del tiempo es estimada por

$$
\hat{\sigma}_{p}^{2}=\sum_{i=1}^{n} \lambda_{i} \hat{\gamma}_{i p}+m
$$

con $p$ el periodo de tiempo donde se quiere hacer la predicción del valor faltante, $\hat{\gamma}_{i p}$ la función de semivarianza estimada $\gamma(h ; \hat{\theta})$ (por mínimos cuadrados o máxima verosimilitud) evaluada para la distancia $h=\left|t_{i}-t_{p}\right|$, y $m$ es el multiplicador de Lagrange considerado para la restricción de insesgadez.

\section{Estudio de simulación}

Con el propósito de comparar el efecto de los métodos de estimación geoestadística en la predicción de datos faltantes en series temporales, se realizó un ejercicio de simulación con series que siguen modelos autorregresivos. Aunque la teoría geoestadística puede ser aplicada a procesos estocásticos no estacionarios en la media, solo se considera, por simplicidad, el caso de media constante. En el caso de media no constante bastaría hacer una diferenciación regular para estacionarizar la serie. Usando la función arima.sim de R [13] se simularon 100 series de tiempo de tamaño 100 (periodos de tiempo) estacionarias como realizaciones de procesos autorregresivos de orden 1, 2 y 3 definidos, respectivamente, por los modelos

$$
\begin{aligned}
& \tilde{Z}(t)=0,8 \tilde{Z}(t-1)+a(t), \\
& \tilde{Z}(t)=0,7 \tilde{Z}(t-1)+0,2 \tilde{Z}(t-2)+a(t), \\
& \tilde{Z}(t)=0,6 \tilde{Z}(t-1)+0,2 \tilde{Z}(t-2)+0,02 \tilde{Z}(t-3)+a(t) .
\end{aligned}
$$



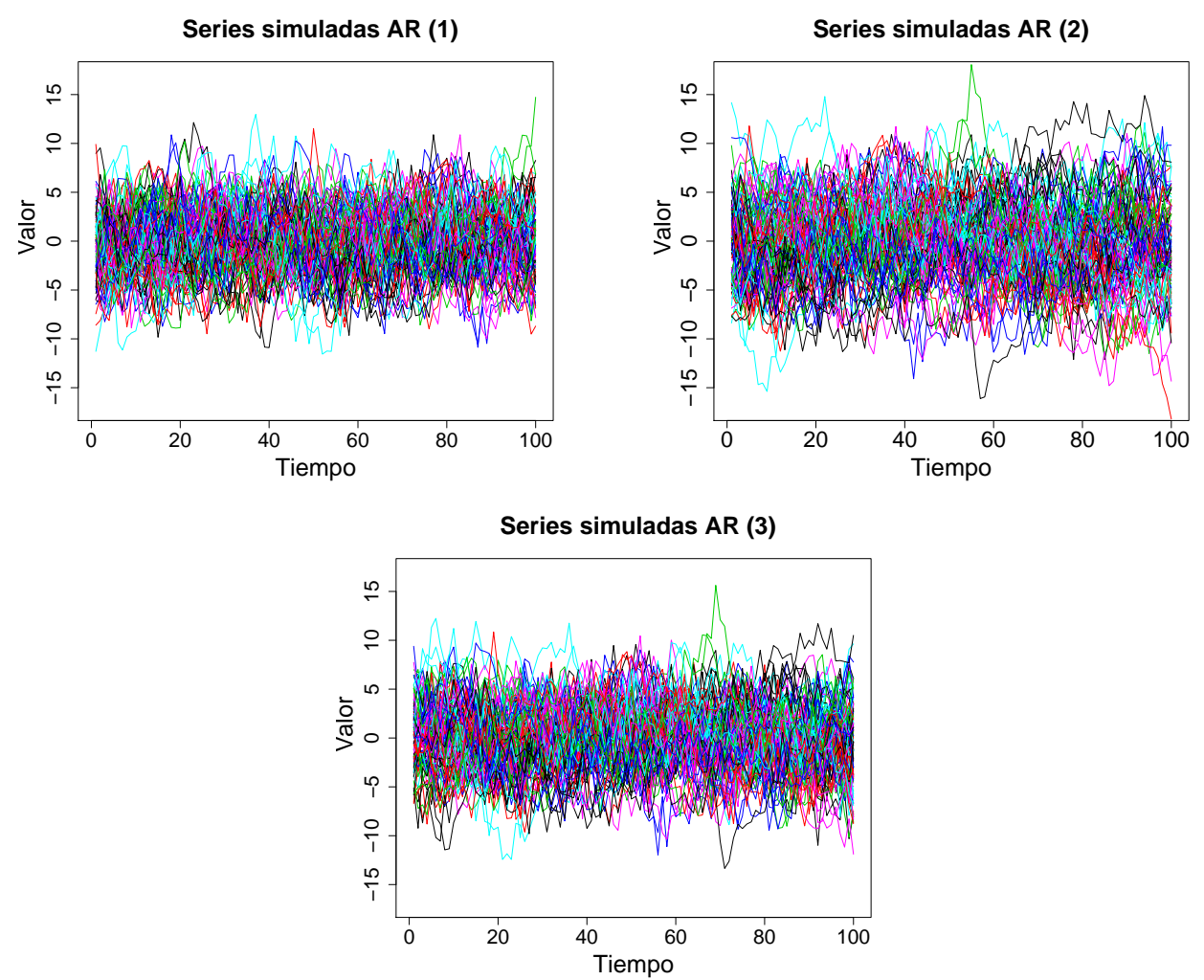

Figura 1. Arriba izquierda: cien series de tiempo simuladas de un modelo AR (1) con $\phi_{1}=0,8$. Arriba derecha: cien series de tiempo simuladas de un modelo $\mathrm{AR}(2)$ con $\phi_{1}=0,7, \phi_{2}=0,2$; Abajo: cien series de tiempo simuladas de un modelo AR (3) con coeficientes $\phi_{1}=0,6, \phi_{2}=0,2$ y $\phi_{3}=0,02$.

En todos los casos se considera $\mu=0$ y $\sigma_{a}^{2}=4$. Nótese que se escogen modelos AR con coeficientes positivos. Esto hace que la función de autocorrelación simple (FAS) decrezca exponencialmente [1] y permite establecer a través de regresiones lineales la relación entre ella misma y la función de semivariograma exponencial de parámetros $\sigma^{2}$ y $\phi$ (ecuación 10). Los valores de los coeficientes en los modelos dados en las ecuaciones (17), (18) y (19) fueron asignados arbitrariamente, pero teniendo en cuenta que ellos garantizaran las condición de estacionariedad. Las series simuladas se muestran en la Figura 1. Puede observarse que las series son estacionarias (media cero y varianza constante).

En primer lugar en la sección 4.1 se presentan los resultados referentes a la simulación de las series de tiempo con modelo AR (1) (ecuación 17). Posteriormente en la sección 4.2 se muestran los correspondientes a los modelos AR (2) y AR (3).

\subsection{Simulación de un proceso $A R(1)$}

A partir de la expresión (4) se calculó la FAS del proceso (círculos en el panel superior izquierdo de la Figura 2) para rezagos $h=0,1,2, \cdots, 49$. De la ecuación (2) se deduce que $\sigma^{2}=11,11$. A partir de la función de correlación se derivan, usando las relaciones dadas en (9), la correspondientes funciones de covarianza $C(h)$ y semivariograma $\gamma(h)$ (para

Vol. 35, No. 1, 2017] 

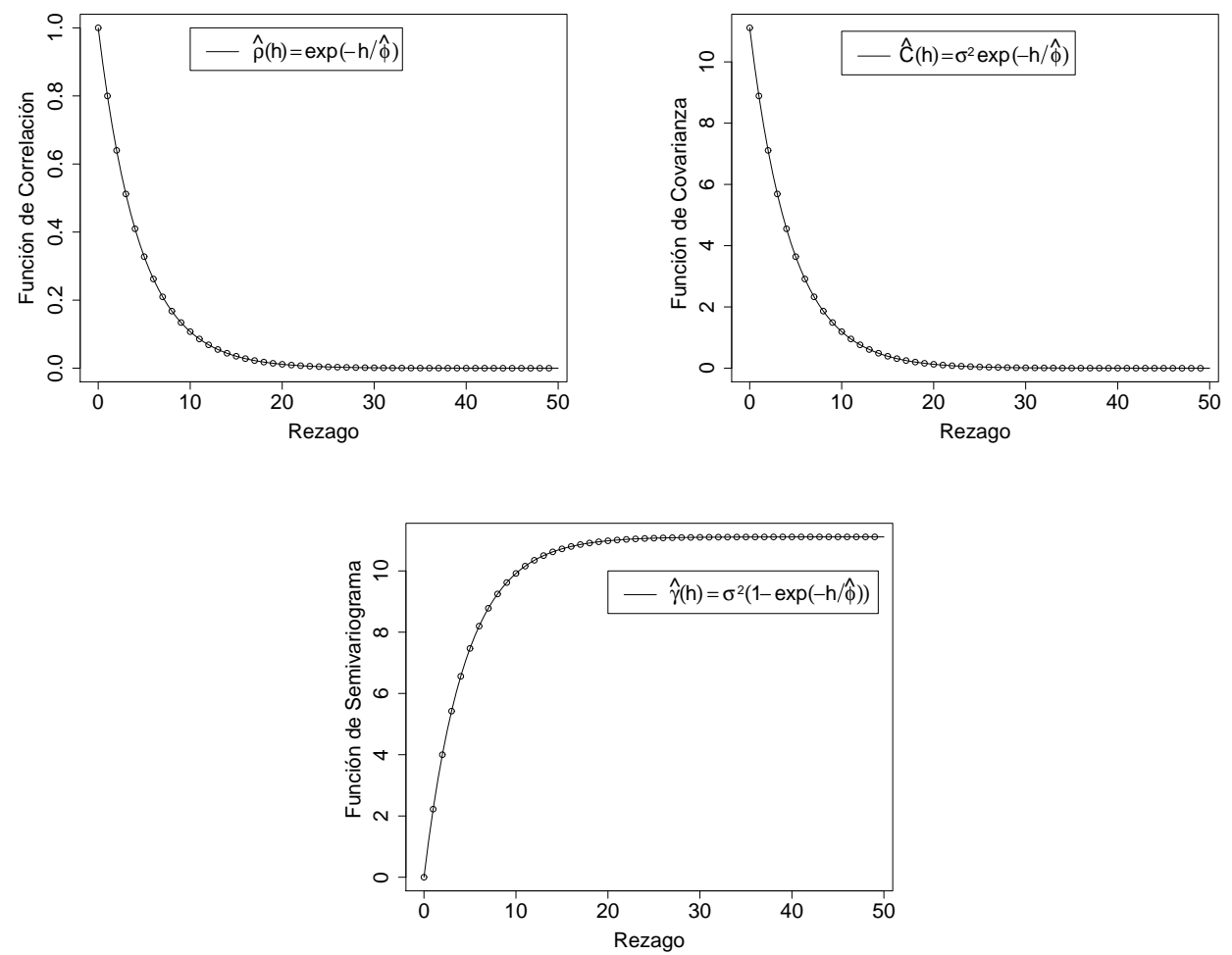

Figura 2. Arriba izquierda: Función teórica de correlación de un $\mathrm{AR}(1)$ con coeficiente $\phi_{1}=0,8$ (círculos) y ajuste de un modelo exponencial (línea continua). Arriba derecha: Función teórica de covarianza de un AR (1) con coeficiente $\phi_{1}=0,8$ (círculos) y ajuste de un modelo exponencial (línea continua). Abajo: Función teórica de semivariograma de un AR (1) con coeficiente $\phi_{1}=0,8$ (círculos) y ajuste de un modelo exponencial (línea continua).

$h=0,1,2,3, \cdots)$. Los gráficos de estas tres funciones se presentan en la Figura 2. En cada caso los círculos corresponden a los valores teóricos del modelo AR (1) considerado y las curvas (líneas continuas) al ajuste de modelos exponenciales definidos como

$$
\begin{aligned}
& \hat{\rho}(h)=\exp \left(\frac{-h}{\hat{\phi}}\right), \\
& \hat{C}(h)=\sigma^{2} \exp \left(\frac{-h}{\hat{\phi}}\right), \\
& \hat{\gamma}(h)=\sigma^{2}\left(1-\exp \left(\frac{-h}{\hat{\phi}}\right)\right) .
\end{aligned}
$$

Para obtener $\hat{\phi}$ se llevó a cabo un análisis de regresión simple entre $h$ y $\rho(h)$ (ecuación 4). En concreto se estimó el modelo lineal sin intercepto $-\log (\rho(h))=\frac{1}{\phi} h+e, h=$ $1,2,3, \cdots, 49$. Se obtuvo en este caso un ajuste perfecto, $R^{2}=1$. Los cálculos fueron realizados mediante la función $1 \mathrm{~m}$ de $\mathrm{R}[13]$. El valor estimado fue $\hat{\phi}=4,5$. Aleatoriamente se sacaron 10 datos de cada una de las 100 series para obtener un total de 1000 valores 
faltantes (panel izquierdo, Figura 3). Con base en los datos de cada una de las series simuladas (después de excluidos los 10 datos de cada una de ellas) se estimaron modelos de semivariograma usando los métodos de MC y MV expuestos en las secciones 3.1 y 3.2, respectivamente. En todos los casos se empleó un modelo de semivariograma exponencial (siendo coherente con el modelo de semivariograma teórico del $\mathrm{AR}(1)$ dado en la Figura 2). La estimación de los semivariogramas se hizo con las funciones variofit (MC) y likfit (MV) de geoR [14]. Ambos procesos acuden a métodos numéricos, y por ello hay que dar valores iniciales (muy razonables) de los parámetros $\sigma^{2}$ y $\phi$ del modelo de semivariograma exponencial. Para esto se tuvo en cuenta el proceso de estimación de $\phi$ arriba mencionado. Con todas las series simuladas se dio como punto de arranque $\sigma^{2}=11,11$ y $\phi=4,5$ (el calculado con la regresión). Los semivariogramas estimados para las cien series simuladas tanto por MC como por MV se presentan en la Figura 4. Una revisión de las estimaciones muestra que en el caso de MC hay algunas que no siguen el patrón común del modelo teórico (línea continua). Estas corresponden a aquellas con valores $\hat{\phi}_{M C}=0$ y $\hat{\phi}_{M C}=44$ (Cuadro 2). De acuerdo con esta figura, el método MV es más apropiado porque permite tener menor dispersión en los estimaciones de los parámetros, particularmente en las del rango.
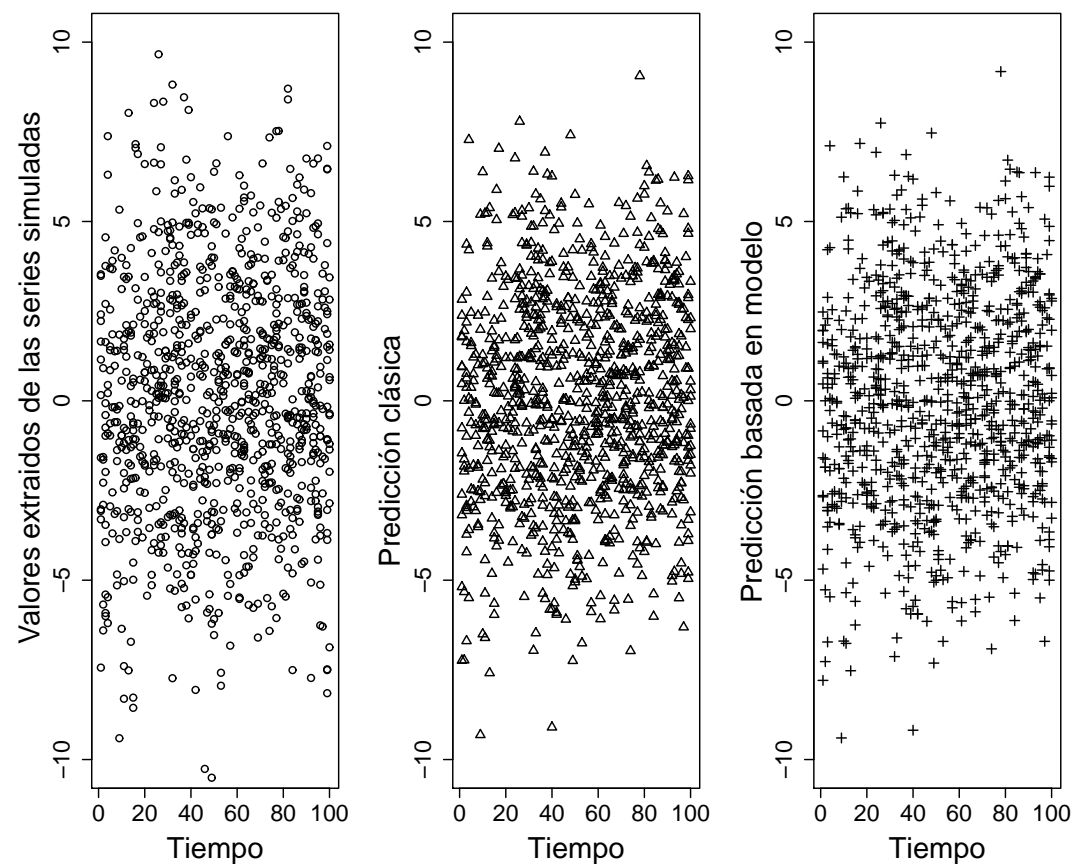

Figura 3. Valores sacados de las series simuladas (izquierda), predicciones por geoestadística clásica (centro) y predicciones por geoestadística basada en modelo (derecha).

Los valores eliminados de las series fueron predichos usando la metodología geoestadística expuesta en la Sección 3. Se aplicó el método Kriging (Sección 3.3) para obtener la predicción de cada uno de los 1000 valores excluidos cuando el semivariograma se estimó por MC (panel central, Figura 3) y por máxima verosimilitud (panel derecho, Figura 3).

Vol. 35, No. 1, 2017] 

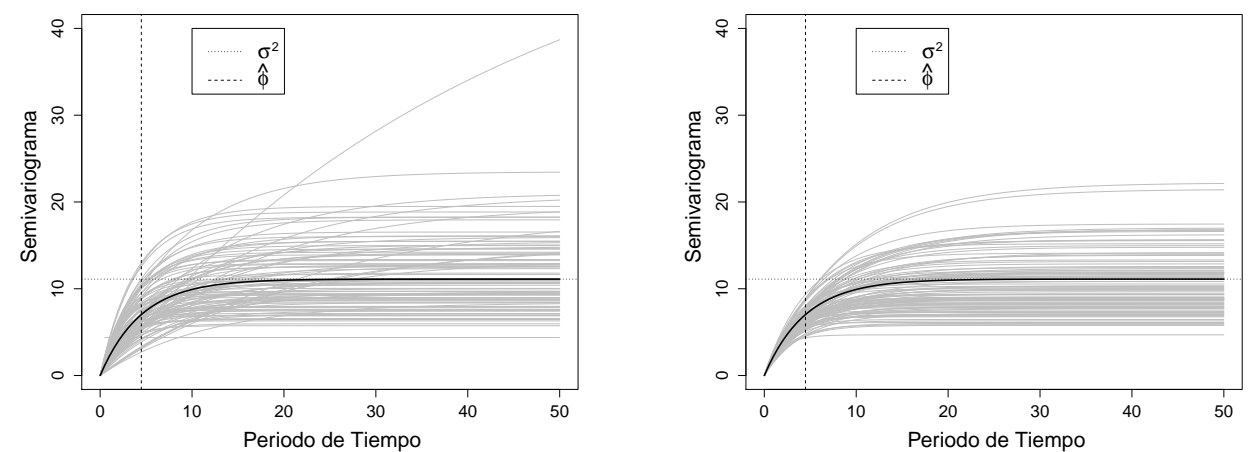

Figura 4. Cien estimaciones (líneas grises) de la función de semivariograma por mínimos cuadrados (izquierda) y por máxima verosimilitud (derecha). La línea punteada horizontal correesponde a la varianza teórica del AR (1) de estudio, la línea punteada vertical al valor $\hat{\phi}$ que fue estimado a partir de la función de correlación teórica de este mismo y la línea negra continua al semivariograma teórico que se obtiene a partir de $\sigma^{2}$ y $\hat{\phi}$.

En cada caso a través de la ecuación (16) se estimó también la correspondiente varianza de predicción. La primera impresión al comparar los tres diagramas de dispersión de la Figura 3 es que estos son muy similares, lo cual indica empíricamente que los dos métodos producen predicciones muy razonables de los valores faltantes y que no hay una marcada diferencia entre ellos.

En total se dispone de 1000 observaciones faltantes (10 dentro de cada una de las 100 series), de 1000 predicciones Kriging de cada una de estas y de 1000 varianzas de predicción. Para hacer una comparación numérica y más detallada de los resultados obtenidos por los dos métodos se calcularon estadísticas descriptivas de las suma de cuadrados de los errores $(S C E)$ y de las varianzas de predicción (Cuadro 1). Las estadísticas de la SCE se obtuvieron con base en las sumas de cuadrados del error dentro de cada serie $S C E_{j}=\sum_{i=1}^{10}\left(O_{i j}-P_{i j}\right)^{2}, j=1, \cdots, 100$, con $O_{i j}$ y $P_{i j}$ correspondientes a la i-ésima observación faltante en la j-ésima serie y a la i-ésima predicción en la j-ésima serie, respectivamente. Las estadísticas de las varianzas de predicción se calcularon con las 1000 estimaciones.

Los valores calculados de las SCE de los dos métodos (Cuadro 1) son muy similares corroborando lo que se muestra en la Figura 3. Un test de Wilcoxon [8] indica que no hay diferencias significativas entre las distribuciones de las $\mathrm{SCE}$ (valor $\mathrm{P}=0,8345$ ). En lo referente a las varianzas de predicción aunque los valores por ambos métodos lucen similares (Cuadro 1), un test de Wilcoxon establece que hay diferencias entre estos (valor $\mathrm{P}<0,0001$ ), lo cual según los valores de media, mediana y máximo de las varianzas de predicción (Cuadro 1) indicaría que el método MV es mejor en este caso, puesto que daría intervalos de confianza (IC) de predicción de menor amplitud. La varianza de predicción (ecuación 16) depende de los valores de la función de semivariograma para las distancias entre los puntos de observación y el sitio de predicción. En otras palabras está en función de las estimaciones de la varianza $\left(\sigma^{2}\right)$ y del rango $(\phi)$. El cálculo de la varianza de predicción no requiere de valores, solo del conocimiento del modelo de semivariograma. Por ello resulta fácil esquematizar dicha relación. Basta fijar un tipo de

[Revista Integración, temas de matemáticas 


\begin{tabular}{|l|c|c|c|c|}
\hline Estadística & SCMC & SCMV & VPMC & VPMV \\
\hline \hline Mínimo & 0,00 & 0,04 & 0,72 & 1,65 \\
Mediana & 14,44 & 14,62 & 2,71 & 2,47 \\
Media & 28,96 & 30,65 & 2,89 & 2,62 \\
Máximo & 152,20 & 156,1 & 11,01 & 8,97 \\
DE & 35,04 & 37,12 & 1,22 & 0,64 \\
DMA & 18,93 & 19,47 & 1,26 & 0,51 \\
\hline
\end{tabular}

Cuadro 1. Estadísticas descriptivas de la suma de cuadrados de los errores de predicción y de las varianzas de predicción obtenidas por MC y MV calculadas con base en las simulaciones del AR (1). SCMC: Suma de cuadrados método mínimos cuadrados; SCMV: suma de cuadrados método máxima verosimilitud; VPMC: Varianza de predicción mínimos cuadrados; VPMV: Varianza de predicción máxima verosimilitud; DE: Desviación estándar; DMA: Desviación mediana absoluta.
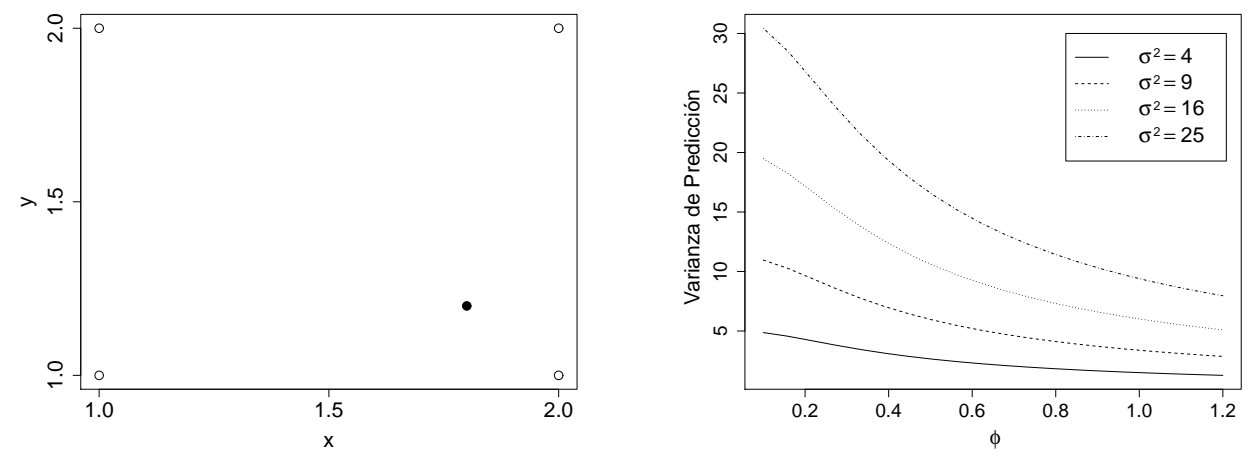

Figura 5. Izquierda: Coordenadas de 4 puntos del plano arbitrariamente escogidos (círculos blancos, $(x, y)=\{(1,1),(1,2),(2,1),(2,2)\})$ y de un sitio de predicción (círculo negro, $(x, y)=(1.8,1.2))$. Derecha: Relación de la varianza de predicción con los párametros $\sigma^{2}=(4,9,16,25)$ y $\phi$ (que toma 20 valores igualmente espaciados entre 0,1 y 1,2 ).

modelo de semivariograma, posibles valores de los parámetros, un enmallado de sitios y un sitio de predicción. Con esto a la mano se puede calcular la ecuación (16). En la Figura 5 se ilustra con un ejemplo simple dicha relación. Supóngase que tiene 4 sitios de observación (círculos blancos en el panel izquierdo) un sitio de predicción (círculo negro del panel izquierdo). Asumiendo un modelo exponencial y tomado 4 valores para la varianza $\left(\sigma^{2}=4,9,16,25\right)$ y 20 valores para $\phi$ en el intervalo $[0.1,1.2]$ se puede observar (panel derecho) que la varianza de predicción disminuye cuando disminuye la varianza del proceso y cuando aumenta el rango, es decir que aun cuando el modelo de semivariograma estimado no se acerque mucho al modelo teórico que lo genera, una subestimación de la varianza o sobreestimación del rango generará una disminución en la varianza de predicción. Por esta razón el criterio de comparación basado en las varianzas de predicción requiere cuidado y no debe hacerse sin tener en cuenta simultáneamente la distribución de los estimaciones $\hat{\phi}$ y $\hat{\sigma}^{2}$.

Los dos procedimientos se comparan también calculando las estadísticas descriptivas de las estimaciones de $\sigma^{2}$ y $\phi$, y particularmente términos del cuadrado medio del error de

Vol. 35, No. 1, 2017] 
estimación a través de $C M E(\hat{\phi})=\sum_{i=1}^{100}\left(\hat{\phi}_{i}-4,5\right)^{2}$ y $C M E\left(\hat{\sigma}^{2}\right)=\sum_{i=1}^{100}\left(\hat{\sigma}_{i}^{2}-11,5\right)^{2}$. Puede observarse en el Cuadro 2 que en general se obtienen mejores resultados con MV. Hay menor CME para el método basado en MV tanto para $\hat{\phi}$ como para $\hat{\sigma}^{2}$. Realizando tests de Wilcoxon se encuentra que no hay diferencias significativas entre las estimaciones de los rangos (valor $\mathrm{P} \simeq 1$ ), pero sí entre las estimaciones de la varianza (valor $\mathrm{P}=0,072$ ). Las distribuciones de ambos parámetros son sesgadas y en el caso de MC tiene varios atípicos. Por eso se considera también la desviación mediana absoluta (DMA) como valor de comparación. En ambos casos con este criterio resulta también mejor el método MV. Los resultados en el Cuadro 2 indican que el método basado en MV arroja estimaciones más cercanas a los parámetros $\phi$ y $\sigma^{2}$. Con base en las distribuciones de $\hat{\phi}$ y $\hat{\sigma}^{2}$ se calcularon los percentiles 5 y 95 y a partir de ellos se obtuvieron IC del $90 \%$ para ambos parámetros por los dos métodos MC y MV (Cuadro 1). Se observa que tanto para $\phi$ como para $\sigma^{2}$ por MV se obtienen IC de estimación de menor amplitud.

De los resultados dados en el Cuadro 1 se dedujo que no hay diferencias entre los dos métodos en términos de las predicciones (SCE estadísticamente iguales) pero que había diferencias entre ellos en los referente a las varianzas de predicción siendo mejor MV. El hecho de que las estadísticas descriptivas y el CME dados en el Cuadro 2 indiquen que con MV se obtienen mejores estimaciones, permite concluir que a pesar de que no hay diferencias en predicciones es preferible usar MV en este caso, puesto que se obtendrán IC de predicción de menor amplitud.

\begin{tabular}{|l|c|c|c|c|}
\hline Estadística & $\hat{\phi}_{M C}$ & $\hat{\phi}_{M V}$ & $\hat{\sigma}_{M C}^{2}$ & $\hat{\sigma}_{M V}^{2}$ \\
\hline \hline Mínimo & 0,00 & 1,7 & 4,37 & 4,68 \\
Mediana & 3,92 & 4,21 & 10,73 & 9,52 \\
Media & 5,59 & 4,39 & 11,98 & 10,55 \\
Máximo & 44,09 & 8,74 & 50,04 & 22,21 \\
DE & 5,57 & 1,56 & 6,03 & 3,45 \\
DMA & 1,81 & 1,51 & 0,62 & 0,49 \\
CME & 31,99 & 2,42 & 0,49 & 0,27 \\
IC 90\% & {$[2.01,14.90]$} & {$[2.33,7.56]$} & {$[6.48,19.49]$} & {$[6.28,16.79]$} \\
\hline
\end{tabular}

Cuadro 2. Estadísticas descriptivas de las 100 estimaciones de la varianza $\left(\sigma^{2}\right)$ y del rango $(\phi)$ calculadas con base en las simulaciones del AR (1). IC $90 \%$ corresponde a intervalos de confianza del $90 \%$ calculados con base en los percentiles 5 y 95 de las estimaciones. DE: Desviación estándar; CME: Cuadrado medio del error; DMA: Desviación mediana absoluta.

\subsection{Simulación de procesos $A R$ (2) y $A R$ (3)}

A continuación se presentan los resultados obtenidos con las simulaciones de los procesos $\operatorname{AR}(2)$ y $\operatorname{AR}(3)$ definidos en las ecuaciones (18) y (19). Empleando la ecuación (6) con $\phi_{1}=0,7 \mathrm{y} \phi_{2}=0,2$ se obtienen los siguientes valores de la función de correlación del 

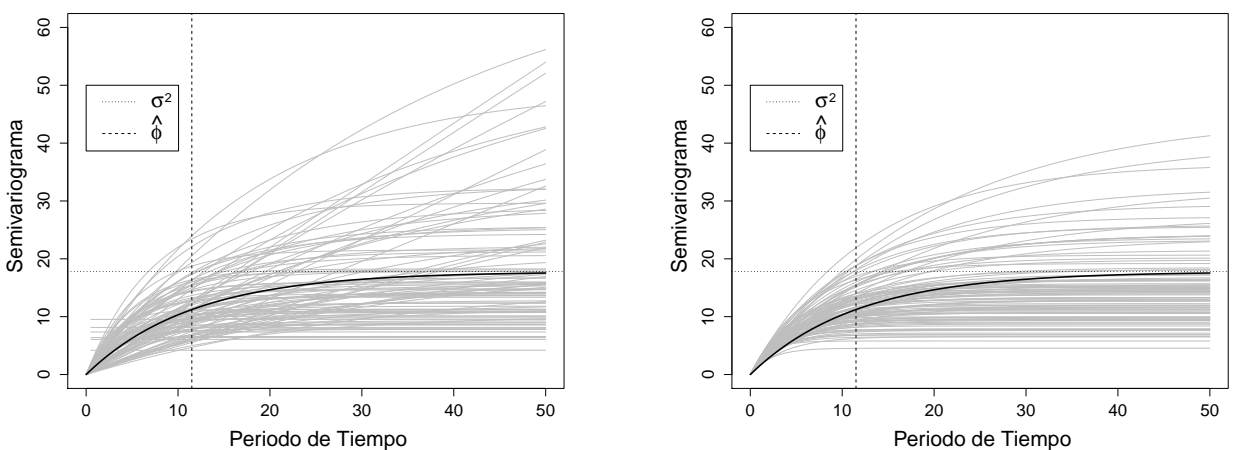

Figura 6. Cien estimaciones (líneas grises) de la función de semivariograma por mínimos cuadrados (izquierda) y por máxima verosimilitud (derecha). La línea punteada horizontal corresponde a la varianza teórica del $\mathrm{AR}(2)$ de estudio, la línea punteada vertical al valor $\hat{\phi}$ que fue estimado a partir de la función de correlación teórica de este mismo y la línea negra continua al semivariograma teórico que se obtiene a partir de $\sigma^{2}$ y $\hat{\phi}$.

proceso AR (2) de estudio:

$$
\begin{aligned}
& \rho(0)=\rho(Z(t), Z(t))=1, \\
& \rho(1)=\rho(Z(t), Z(t-1))=\frac{\phi_{1}}{\left(1-\phi_{2}\right)}=0,875, \\
& \rho(2)=\rho(Z(t), Z(t-2))=\frac{\phi_{1}^{2}}{\left(1-\phi_{2}\right)}+\phi_{2}=0,8125,
\end{aligned}
$$

y para $h \in \mathbb{Z}$, con $h \geq 3$, se usa la forma recursiva

$$
\rho(h)=\rho(Z(t), Z(t-h))=0,7 \rho_{h-1}+0,2 \rho_{h-2} .
$$

De la ecuación (7) se deduce que $\sigma^{2}=17,8$. Siguiendo un procedimiento análogo al descrito con las simulaciones del proceso AR (1) y a partir de los valores de la FAS, y del ajuste de un modelo de regresión (después de transformar a logaritmos) se obtuvo la estimación de $\phi$ para el modelo exponencial. El valor en este caso fue $\hat{\phi}=11,5$. Se simularon nuevamente 100 series y se excluyeron 10 de cada una de ellas. Con cada serie simulada (después de excluidos los 10 datos) se estimaron modelos de semivariograma usando los métodos de MC y MV. En todos los casos se empleó un modelo de semivariograma exponencial con valores iniciales de arranque $\sigma^{2}=17,8$ y $\phi=11,5$ (el calculado con la regresión). Los semivariogramas estimados para las 100 series simuladas tanto por MC como por MV se presentan en la Figura 6. Nuevamente algunos semivariogramas estimados por MC presentan comportamientos atípicos. Algunos estimaciones del rango son iguales a cero (líneas rectas) y algunas son muy altas (por ejemplo $\hat{\phi}_{M C}=312100$, ver Cuadro 4) y obviamente carentes de sentido. Desde un punto de vista gráfico el método MV tiene un mejor comportamiento.

Una vez estimados los modelos de semivariograma y usando Kriging se hicieron predicciones de los 10 valores faltantes en cada serie. A partir de las observaciones y las predicciones se calcularon las SCE (Cuadro 3). Estas, como en el caso del AR (1), no

Vol. 35, No. 1, 2017] 
reflejan diferencias apreciables entre los dos métodos. Un test de Wilcoxon indica que no hay diferencias significativas entre las distribuciones de las SCE (valor $\mathrm{P}=0,6877$ ). En términos de la varianza de predicción los valores hallados por MV son mayores (el test de Wilcoxon da un valor $\mathrm{P}<0,0001$ ). Este resultado es contario al encontrado en el AR (1) y sugeriría como mejor opción el método de MC. No obstante, al observar los resultados de las estadísticas descriptivas de las estimaciones $\hat{\phi}$ y $\hat{\sigma}^{2}$, y en particular el CME (Cuadro 4), se concluye que por MV se obtienen estimaciones con menor sesgo.

Por consiguiente, aunque con MC se pueden obtener IC de predicción de menor amplitud (porque las estimaciones del rango son altas) estos no son apropiados. La varianza de predicción en este caso se ve reducida por el sesgo considerable en las estimaciones del rango. En resumen, teniendo en cuenta lo anterior (menor sesgo en las estimaciones de los parámetros), puede decirse que los resultados de las simulaciones del AR (2), al igual que en el caso de las del AR (1), indican que es mejor el método de MV.

\begin{tabular}{|l|c|c|c|c|}
\hline Estadística & SCMC & SCMV & VPMC & VPMV \\
\hline \hline Mínimo & 0,02 & 0,08 & 0,48 & 1,61 \\
Mediana & 12,02 & 12,11 & 2,05 & 2,42 \\
Media & 33,98 & 32,00 & 2,34 & 2,56 \\
Máximo & 174,50 & 175,50 & 9,63 & 8,73 \\
Suma & 3398 & 3199 & 2342 & 2563 \\
DE & 47,57 & 43,40 & 1,63 & 0,67 \\
DMA & 16,84 & 17,37 & 1,26 & 0,51 \\
\hline
\end{tabular}

Cuadro 3. Estadísticas descriptivas de la suma de cuadrados de los errores de predicción y de las varianzas de predicción obtenidas por ambos métodos. SCMC: suma de cuadrados mínimos cuadrados; SCMV: suma de cuadrados máxima verosimilitud; VPMC: varianza de predicción mínimos cuadrados; VPMV: varianza de predicción máxima verosimilitud; DE: desviación estándar; DMA: desviación mediana absoluta.

\begin{tabular}{|l|c|c|c|c|}
\hline Estadística & $\hat{\phi}_{M C}$ & $\hat{\phi}_{M V}$ & $\hat{\sigma}_{M C}^{2}$ & $\hat{\sigma}_{M V}^{2}$ \\
\hline \hline Mínimo & 0,00 & 1,80 & 4,19 & 4,55 \\
Mediana & 8,77 & 5,93 & 16,45 & 13,55 \\
Media & 3472 & 6,62 & 2321 & 15,19 \\
Máximo & 312100 & 19,59 & 203400 & 44,76 \\
DE & 31335 & 3,53 & 20458 & 7,44 \\
CME & $9,8 \times 10^{8}$ & 36,29 & 2246 & 0,9347 \\
DMA & 7,19 & 2,85 & 1,13 & 0,74 \\
IC 90\% & {$[0.00,130.27]$} & {$[2.87,13.90]$} & {$[7.78,79.24]$} & {$[6.89,29.56]$} \\
\hline
\end{tabular}

Cuadro 4. Estadísticas descriptivas de las 100 estimaciones de la varianza $\left(\sigma^{2}\right)$ y del rango $(\phi)$. IC $90 \%$ corresponde a intervalos de confianza del $90 \%$ calculados con base en los percentiles 5 y 95 de las estimaciones. DE: desviación estándar; CME: cuadrado medio del error; DMA: desviación mediana absoluta.

Finalmente, dentro de esta sección se mencionan muy brevemente los resultados obtenidos 
con las simulaciones del proceso AR (3) definido en la ecuación (19). Las conclusiones generales a las que se llega con estas son análogas a las descritas con los procesos AR (1) y AR (2), y por ello no se incluyen tablas adicionales. Un test de Wilcoxon indica que no hay diferencias en las estimaciones de los rangos (valor $\mathrm{P} \simeq 1$ ) y que las varianzas son significativamente diferentes (valor $\mathrm{P}=0,0429$ ). En general las medidas descriptivas de los rangos sugieren, como en el AR (1) y en AR (2), que con MV se obtienen mejores resultados (estos son similares a los de la simulación del AR (2) presentados en el Cuadro 4), en el sentido de que se obtiene menor CME, menor DMA y los intervalos de confianza del $90 \%$ tienen menor amplitud.

Como en las simulaciones de los procesos AR (1) y AR (2) un test de Wilcoxon indica que no hay diferencias entre las $\mathrm{SCE}$ (valor $\mathrm{P}=0,6779$ ) y que por consiguiente los dos métodos permiten obtener predicciones muy similares. Las varianzas de predicción son ligeramente mayores cuando se usa MV. Esto es consecuencia de la sobreestimación de los rangos que se da cuando se usa MC, y por ello, como se mencionó anteriormente, esto no debe considerarse como criterio de selección.

\section{Aplicación}

A manera de ilustración con datos reales, se hace predicción de datos faltantes en la serie de tiempo correspondiente a datos quincenales (entre enero de 1987 y marzo de 1991) de la variable transparencia del agua, medidos por medio de visibilidad del disco de Secchi [11] en la estación Canal de Caño Grande (CCG) del sistema lagunar Ciénaga Grande de Santa Marta (CGSM) ubicado en la costa norte de Colombia. La serie temporal (Figura 7, panel izquierdo), presenta 16 observaciones faltantes a lo largo del periodo de tiempo considerado (Cuadro 5). Dichas observaciones fueron predichas usando Kriging ordinario y empleando MC y MV en la fase de estimación de la función de semivariograma.

En primera instancia, se calculó el semivariograma experimental (ecuación 11) y se ajustó un modelo exponencial $\gamma(h)=\tau^{2}+\sigma^{2}(1-\exp (-h / \phi)$ por MC (Figura 8). Se ensayaron varios modelos y se escogió el exponencial porque este produjo la menor suma de cuadrados de los errores. Los valores de los parámetros estimados son dados en el Cuadro 6. Se usó la función variofit de la librería geoR [14] para hacer la estimación. De acuerdo con el valor del rango se concluye que hay influencia temporal entre observaciones separadas hasta 4 quincenas (alrededor de dos meses). Se detectó también la presencia de efecto nugget, debido a la fuerte tasa de cambio en el semivariograma para distancias cercanas al origen. Esto último, en el caso de estudios espaciales, está asociado a la escala de medida o a errores de medición de la variable. Dentro del contexto de series temporales, podría estar referido a la varianza en cada punto del tiempo [15]. Un segundo camino fue basado en la estimación del semivariograma empleando MV. Se asumió normalidad multivariada (es decir que se asume que el proceso estocástico que genera la serie es gaussiano) y un modelo de semivariograma exponencial para definir la matriz de varianzas y covarianzas y se realizó la maximización de la función log-verosímil resultante empleando la función likfit de la libería geoR [14]. Las estimaciones son dadas en el Cuadro 6. La diferencia esencial entre el modelo estimado por esta vía y el estimado por el camino clásico fue la no detección de efecto nugget. El rango estimado fue nuevamente de 4 quincenas.

Con base en los modelos de semivariograma hallados, se hizo estimación de los parámetros $\lambda_{i}$ dados en el predictor (15) para obtener con ellos las predicciones de cada uno de los

Vol. 35, No. 1, 2017] 


\begin{tabular}{|l|r|c|c|c|c|}
\hline OF & Fecha & PMC & EEPMC & PMV & EEPMV \\
\hline \hline 1 & Septiembre 01 1987 & 47,3 & 8,5 & 45,1 & 7,1 \\
2 & Septiembre 15 1987 & 47,6 & 8,5 & 48,0 & 7,1 \\
3 & Diciembre 15 1987 & 41,7 & 8,1 & 41,7 & 6,2 \\
4 & Enero 15 1988 & 37,0 & 8,1 & 35,3 & 6,2 \\
5 & Marzo 15 1988 & 34,3 & 8,1 & 33,0 & 6,2 \\
6 & Agosto 01 1988 & 48,0 & 8,5 & 50,1 & 7,1 \\
7 & Agosto 15 1988 & 49,6 & 8,5 & 52,4 & 7,1 \\
8 & Noviembre 15 1988 & 45,1 & 8,5 & 46,1 & 7,1 \\
9 & Diciembre 01 1988 & 43,6 & 8,5 & 42,2 & 7,1 \\
10 & Abril 01 1989 & 55,0 & 8,1 & 57,2 & 6,2 \\
11 & Febrero 01 1990 & 56,6 & 8,1 & 57,2 & 6,2 \\
12 & Febrero 15 1990 & 47,8 & 8,5 & 45,5 & 7,1 \\
13 & Mayo 15 1990 & 46,5 & 8,5 & 42,9 & 7,1 \\
14 & Julio 01 1990 & 57,0 & 8,1 & 59,6 & 6,2 \\
15 & Julio 15 1990 & 57,4 & 8,1 & 59,6 & 6,2 \\
16 & Marzo 01 1991 & 62,8 & 9,1 & 65,0 & 7,9 \\
\hline
\end{tabular}

Cuadro 5. Fecha de datos faltantes con su respectiva predicción y error estándar de predicción. $\mathrm{OF}=$ Observación faltante; $\mathrm{PMC}=$ Predicción Kriging estimando por mínimos cuadrados $(\mathrm{MC})$; $\mathrm{EEPMC}=$ Error estándar de predicción estimando por $\mathrm{MC}$; PMV= Predicción Kriging estimando por máxima verosimilitud (MV); EEPMV= Error estándar de predicción del Kriging, estimando por MV.

\begin{tabular}{|l|r|r|}
\hline Parámetro & MC & MV \\
\hline \hline Efecto nugget $\left(\tau^{2}\right)$ & 34,6 & 0,0 \\
Varianza $\left(\sigma^{2}\right)$ & 94,9 & 159,8 \\
Rango $(\phi)$ & 4,5 & 4,1 \\
\hline
\end{tabular}

Cuadro 6. Parámetros estimados (por mínimos cuadrados y máxima verosimilitud) para un modelo de semivariograma exponencial con base en los datos de la serie temporal de transparencia del agua medida en la estación CCG de la CGSM. MC= método basado en mínimos cuadrados; $\mathrm{MV}=$ método basado en máxima verosimilitud.

16 valores faltantes de la serie (para cada predicción se hace una estimación de $\lambda_{i}, i=$ $1, \cdots, n)$. Empleando $\lambda_{i}$ y la estimación de $m$ se calcula también la varianza de predicción dada en (16). Los resultados de las predicciones y de las estimaciones de los errores estándar de predicción (raíz de la varianza de predicción) son mostrados en el Cuadro 5. En la Figura 7 (panel derecho) se presenta la serie completada (con las predicciones de los valores faltantes) y los intervalos de confianza del $95 \%$ para las predicciones. Estos últimos son obtenidos asumiendo normalidad y calculados con base en los errores estándar del método basado en MV. Al comparar los valores dados en el Cuadro 5 se puede concluir que no hay diferencias grandes entre las predicciones obtenidas por los dos métodos, pero que los errores estándar estimados de predicción son menores cuando se usa geoestadística basada en modelo (MV). Esto permite obtener intervalos de confianza 

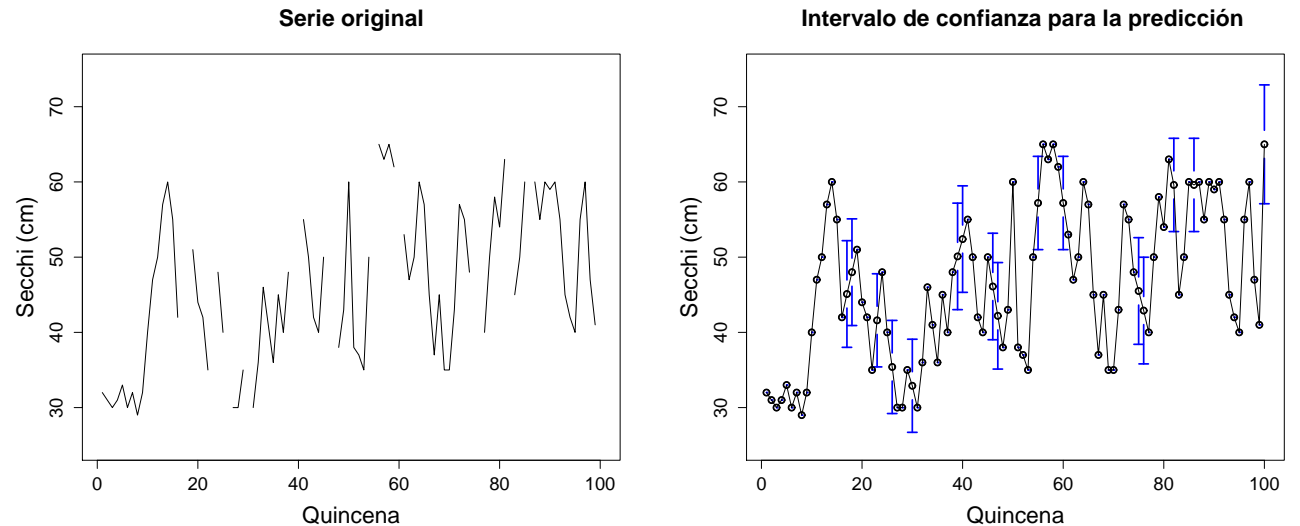

Figura 7. Serie de tiempo, con datos faltantes, correspondiente a mediciones quincenales de la variable transparencia del agua en la estación CCG de la CGSM (panel izquierdo). Serie de tiempo de transparencia del agua con observaciones predichas mediante la técnica Kriging e intervalos de confianza del $95 \%$ para las predicciones (panel derecho).

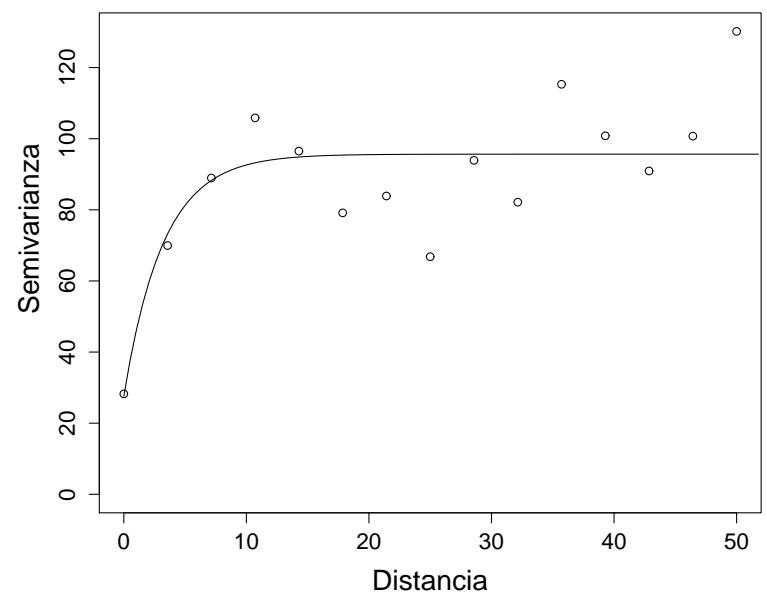

Figura 8. Semivariograma experimental (puntos) y ajuste por mínimos cuadrados ponderados de un modelo exponencial (línea continua) para el mismo, con los datos correspondientes a la serie de tiempo de transparencia del agua en la estación CCG de la CGSM.

de menor amplitud y por ende más útiles desde la perspectiva aplicada.

Como etapa final del análisis se muestran en el Cuadro 7 las estadísticas descriptivas de los residuales obtenidos a partir del método de validación cruzada (VC), es decir que cada observación de la serie es eliminada y posteriormente predicha a través del predictor (15) usando las observaciones restantes y el modelo de semivariograma ajustado. Una comparación de los residuales de los dos procedimientos revela que los mínimos, máximos y medias son similares. Aunque gráficamente no se detectan mayores diferencias entre los métodos cuando se comparan las predicciones de $\mathrm{VC}$ con los valores observados (Figura 9), los coeficientes de determinación $R^{2}$ (correspondientes al ajuste de modelos

Vol. 35, No. 1, 2017] 


\begin{tabular}{|l|r|r|}
\hline Medida & MC & MV \\
\hline \hline Mínimo & $-12,4$ & $-12,5$ \\
Mediana & $-0,7$ & $-0,1$ \\
Media & 0,0 & 0,0 \\
Máximo & 5,1 & 4,2 \\
D.E. & 7,1 & 6,5 \\
\hline
\end{tabular}

Cuadro 7. Medidas descriptivas de los errores de validación cruzada. MC= Residuales del Kriging cuando se estima el variograma por mínimos cuadrados; $\mathrm{MBM}=$ Residuales del Kriging cuando se estima el variograma por máxima verosimilitud. D.E.= Desviación estándar.
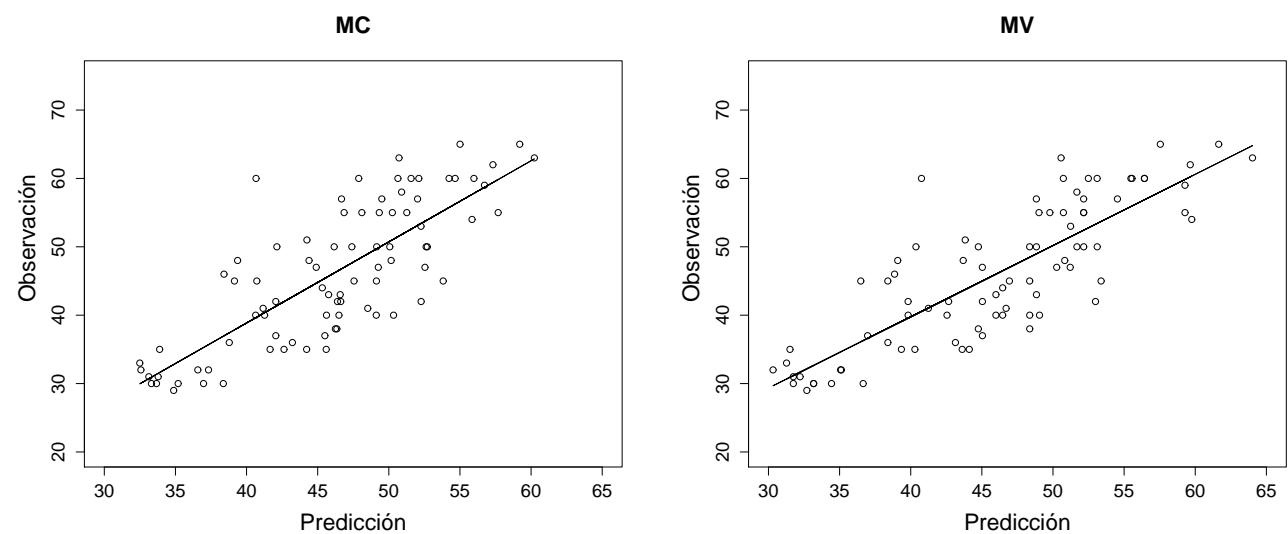

Figura 9. Validación cruzada de predicciones por mínimos cuadrados (izquierda, $R^{2}=0,6222$ ) y máxima verosimilitud (derecha, $R^{2}=0,6786$ ).

de regresión simple entre la respuesta y la predicción), indican que el método basado en MV produce ligeramente mejores resultados. Así mismo, en el Cuadro 7 se puede observar que el procedimiento basado en modelo (MV) permite tener menor variabilidad de los errores de validación cruzada (menor desviación estándar residual), es decir que hay mejor ajuste con el modelo de semivariograma obtenido por esta vía, lo que al final se refleja, como ha sido antes mencionado, en intervalos de confianza más cortos.

\section{Conclusiones y trabajo futuro}

De manera general los resultados de las simulaciones muestran que en lo referente a las predicciones de los valores faltantes los dos caminos de estimación del semivariograma producen resultados equivalentes. Esto no es sorprendente si se tiene en cuenta que, independientemente del modelo de semivariograma escogido, al hacer la predicción Kriging el peso lo tienen las observaciones de los periodos de tiempo más cercanos, que por el proceso de generación de valores faltantes escogido son justamente los contiguos. El efecto de las estimaciones de los parámetros del modelo de semivariograma podría verse en series con largos periodos de vacíos de información y/o no equidistantes en tiempo. Las distribuciones de las estimaciones del rango y de la varianza son asimétricas en ambos 
casos (MC y MV), pero las colas son más pesadas cuando se emplea MC. Esto genera de manera equivocada una disminución en la varianza de predicción, lo cual implica que aunque la amplitud de los intervalos de confianza de las predicciones es menor con MC, es más apropiado considerar los basados en las estimaciones obtenidas por MV.

Como trabajo futuro es de interés determinar la relación entre la función de autocorrelación de otros tipos de series con los modelos válidos de semivariograma (Matérn, Wave, potencial, etc.). También es de interés establecer cómo cambian las distribuciones de los estimadores de los parámetros de la función de semivariograma cuando aumenta el tamaño de las series o el efecto de dichas estimaciones en las predicciones cuando hay muchos periodos de tiempo contiguos con valores faltantes (por ejemplo si hay años completos de información faltante).

\section{Referencias}

[1] Box G., Jenkins G. and Reinsel G., Time Series Analysis: Forecasting and Control, John Wiley \& Sons, New York, 2008.

[2] Cressie N., Statistical for Spatial Data, John Wiley \& Sons, New York, 1993.

[3] De Iaco S., Palma M. and Posa D., "Geostatistics and the Role of Variogram in Time Series Analysis: A Critical Review", en Statistical Methods for Spatial Planning and Monitoring ( ed. Montrone S. and Perchinunno P.), Springer-Verlag, Milan, 2013.

[4] Diggle P. and Ribeiro P., Model-based Geostatistics, Springer-Verlag, New York, 2007.

[5] Dowdall M., Bjorn L., Gerland S. and Rudjord A., "Geostatistical analysis as applied to two environmental radiometric time series", Environmental Monitoring and Assessment 83 (2003), No. 1, 1-16.

[6] Ghahraman B. and Ahmadi F., "Application of geostatistics in time series: Mashhad annual rainfall", Iran Watershed Management Science and Engineering 1 (2007), No. 1, 7-15.

[7] Haslett J., "On the sample variogram and sample autocovariance for non-stationary time series", Journal Royal Statistical Society Series D 46 (1997), No. 4, 475-484.

[8] Hollander T. and Wolfe D., Nonparametric Statistical Methods, John Wiley \& Sons, New York, 1999.

[9] Ljung G., "A note on the estimation of missing values in time series", Comm. Statist. Simulation Comput. 18 (1989), No. 2, 459-465.

[10] Ma Ch., "The use of the variogram in construction of stationary time series models", $J$. Appl. Probab. 41 (2004), No. 4, 1093-1103.

[11] Margalef R., Limnología, Ediciones Omega, Barcelona, 1983.

[12] Peña D. and Tiao G., "A note on likelihood estimation of missing values in time series", Amer. Statist. 45 (1991), No. 3, 212-213.

[13] R Core Team, R: A Language and Environment for Statistical Computing, R Foundation for Statistical Computing, Vienna, 2013.

[14] Ribeiro P. and Diggle P., "geoR: a package for geostatistical analysis", R-NEWS 1 (2001), No. $2,15-18$.

Vol. 35, No. 1, 2017] 
[15] Rouhani S. and Myers D., "Problems in space time Kriging of geohidrological data", Mathematical Geology 5 (1990), No. 22, 611-623.

[16] Schabenberger O. and Gotway C., Statistical Methods for Spatial Data Analysis, Chapman \& Hall, Boca Ratón, 2005. 